\title{
Estudo da Distribuição de Poluentes em Superfícies Urbanas
}

\author{
Maria do Carmo Cauduro Gastaldini, Ana Roberta Victoria Silva \\ Departamento de Engenharia Sanitária e Ambiental - Centro de Tecnologia - Universidade Federal de Santa Maria \\ mcarmocg@gmail.com, anarobvs@yahoo.com.br.
}

Recebido: 29/06/11 - revisado: 27/09/11 - aceito: 06/07/12

\section{RESUMO}

O trabalho visa quantificar poluentes ligados a diversos tamanhos de sedimentos em superfícies impermeáveis. Foram realizadas coletas de sedimento seco em duas ruas e monitoramento hidrossedimentológico na saída da canalização de uma. As amostras de sedimento seco dos dias anteriores à precipitação, bem como as de sedimento ligado ao escoamento superficial, durante a precipitação, foram separadas em 4 faixas granulométricas $(\leq 63 \mu \mathrm{m}, 63-250 \mu \mathrm{m}, 250-500 \mu \mathrm{m}$ e $\geq 500 \mu \mathrm{m})$. Para cada faixa granulométrica foi avaliada a carga de poluição orgânica, de nutrientes e de metais pesados. As amostras de metais pesados apresentaram maiores concentrações na granulometria menor ou igual que 63 4 m. Nas amostras de sedimento seco, as maiores cargas de DQO e fosfato foram encontradas na granulometria maior ou igual que 500um. A amônia e o nitrato, chumbo e zinco estão associados à faixa granulométrica de 63-250um.

Palavras-chave: sedimentos, metais pesados, nutrientes, poluição difusa.

\section{INTRODUÇÃO}

O crescimento populacional e o conseqüente processo de urbanização acelerado e sem planejamento adequado causa efeitos nocivos ao meio ambiente. O desenvolvimento urbano, além de causar alterações no ciclo hidrológico, devido ao aumento de áreas impermeáveis, redução de infiltração no solo e aumento do escoamento superficial; provoca um aumento na quantidade de poluentes devido a fatores antropogênicos tais como uso do solo, atividades locais, volume de tráfego na região, entre outros.

Os principais poluentes carreados pela drenagem urbana são sedimentos, nutrientes, matéria orgânica, bactérias, metais pesados, hidrocarbonetos, pesticidas.

Segundo Carvalho (2000), os sedimentos não são somente um dos maiores poluentes da água, mas também servem como catalisadores, carreadores e fixadores para outros agentes poluidores. Produtos químicos são assimilados sobre e dentro das partículas de sedimento. Trocas iônicas podem ocorrer entre o soluto e o sedimento. As partículas de sedimento agem como potencializadores dos produtos gerados por agentes químicos decorrentes do lixo, resíduos tóxicos, nutrientes, bactérias patogênicas, vírus, etc.
Vaze e Chiew $(2002,2004)$ determinaram a carga de nutrientes associada com diferentes tamanhos de partículas dos poluentes superficiais secos e de amostras coletadas do escoamento superficial de uma rodovia urbana. Concluíram que a totalidade do nitrogênio e do fósforo particulado em amostras do escoamento superficial está unida a sedimentos de tamanhos entre 11 e $150 \mu \mathrm{m}$, portanto, para remover fósforo e nitrogênio o tratamento deve remover partículas até $11 \mu \mathrm{m}$.

Deletic e Orr (2005) amostraram sedimentos em rodovia urbana em Aberdeen (Escócia) por meio do "método úmido", aspirando amostras após a lavagem de superfícies da rodovia. Analisaram concentrações de metais pesados e nutrientes. As concentrações de metais pesados foram maiores na fração de tamanho de partículas menores do que $63 \mu \mathrm{m}$.

Egodawata e Goonetilleke (2006) caracterizaram poluentes acumulados na superfície de ruas de ocupação residencial na Austrália. Análise dos parâmetros da qualidade revelou que uma grande fração de poluentes está associada com a fração de partículas mais finas.

Poleto e Merten (2007) avaliaram poluentes encontrados nos sedimentos de rua e em amostras de sedimento de rio, em bacia urbana na cidade de Porto Alegre. Constataram que as concentrações desses elementos variam temporalmente durante o evento de chuva, devido ao carreamento de sedi- 
mentos depositados nas ruas com altas concentrações de metais pesados, que relacionam-se com o tráfego de veículos e atividades antropogênicas.

Push et al. (2007) determinaram cargas de metais em fontes difusas de poluição urbana no município de Curitiba. Estimaram cargas de $0,00129,0,106,0,128$ e 3,34 kg.ha ${ }^{-1} \cdot$ ano $^{-1}$ para cádmio, cobre, chumbo e zinco, respectivamente, nas águas do escoamento pluvial urbano.

Menezes e Rosso (2007) avaliaram a qualidade da água pluvial, correlacionando aos poluentes originados pelo tráfego de veículos automotores, para o Túnel Rebouças, no município do Rio de Janeiro. Concluíram que as concentrações de nitrogênio e fósforo não estão ligadas diretamente ao desgaste dos veículos na via e sim a outras origens como emissões atmosféricas dos veículos, deposições das precipitações de chuvas.

Conclui-se, portanto, que quantidades significativas de poluentes do escoamento superficial urbano são transportadas como contaminantes ligados aos sedimentos. O conhecimento da quantidade de poluentes ligada a tamanhos de sedimentos é importante, para que o projeto de estruturas de tratamento possa remover a maioria dos sedimentos poluídos.

Este trabalho visa quantificar os poluentes ligados às diversas faixas granulométricas dos sedimentos provenientes de superfícies impermeáveis de áreas urbanas e dos poluentes provenientes das amostras de água da microdrenagem.

\section{METODOLOGIA}

\section{Área de estudo}

A região de estudo encontra-se inserida na bacia hidrográfica do Cancela, situada entre as coordenadas geográficas $53^{\circ} 48^{\prime} 44^{\prime \prime}$ e $53^{\circ} 47^{\prime} 12^{\prime \prime}$ de longitude oeste e $29^{\circ} 43^{\prime} 02^{\prime \prime}$ e $29^{\circ} 41^{\prime} 31^{\prime \prime}$ de latitude sul, no município de Santa Maria-RS. A bacia possui área de $4,95 \mathrm{~km}^{2}$, população de aproximadamente 18.000 habitantes, em alguns trechos apresenta sistema de esgotamento das águas pluviais e residuárias do tipo separador parcial.

Foram selecionadas duas ruas localizadas na porção sudoeste da bacia, a rua Marquês do Herval e a rua Rigoberto Duarte, que foram monitoradas ao longo da sarjeta.

As ruas em questão possuem pavimento impermeável (asfalto) e coleta semanal de lixo, porém desprovêem de varrição. Os poluentes, que nela se depositam, são carreados pela chuva diretamente para as bocas de lobo seguindo para o arroio Cancela. A região é predominantemente residencial com presença de alguns estabelecimentos comerciais.

$\mathrm{O}$ monitoramento de qualidade da água e hidrossedimentológico foi realizado através de uma estação pluviométrica e uma estação fluviométrica, ambas monitoradas pelo grupo GHIDROS, localizados na figura 1. A figura 1 ilustra, também, a área de contribuição à canalização coletora das águas pluviais provenientes da rua Marquês do Herval, onde foi realizado monitoramento hidrossedimentológico.

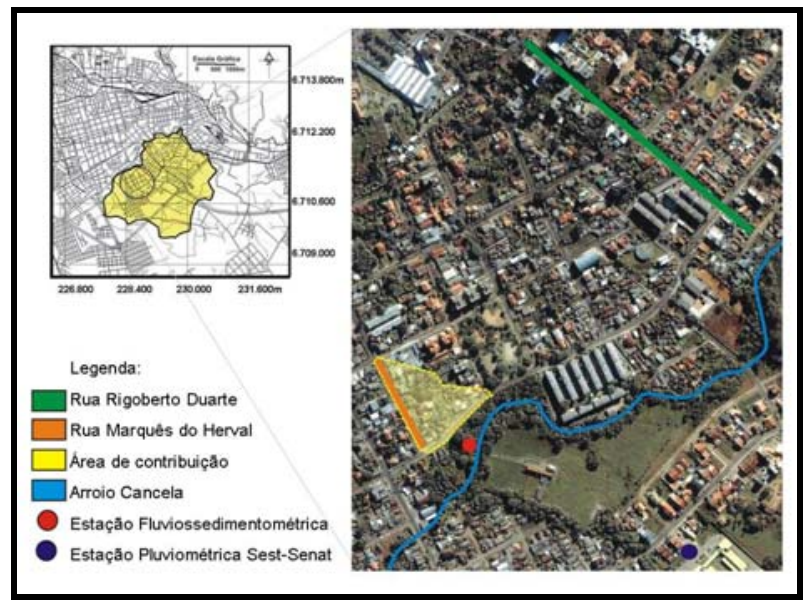

Figura 1 - Localização da área de estudo com as estações de monitoramento.

\section{Amostragem dos sedimentos secos}

Foram realizadas coletas de sedimento seco nas ruas Marquês do Herval e Rigoberto Duarte, a fim de obter dados comparativos em relação à acumulação de poluentes nos sedimentos de superfícies impermeáveis. Na rua Marquês do Herval foi realizado monitoramento hidrossedimentológico na saída da canalização coletora das águas pluviais provenientes desta rua.

Os dados pluviométricos foram obtidos da estação Sest-Senat, localizada próximo às ruas do estudo, monitorada pelo grupo GHIDROS, figura 1 .

Os dados do monitoramento fluviográfico foram obtidos da estação Tamandaí, figura 1. A estação é composta por registrador de nível digital e uma calha de fundo plano, implantados por Dotto (2006). A área de contribuição da drenagem é de $0,012 \mathrm{Km}^{2}$ (GOMES, 2008).

Os sedimentos secos foram coletados ao longo das sarjetas da rua Marquês do Herval durante três períodos, e Rigoberto Duarte durante dois pe- 


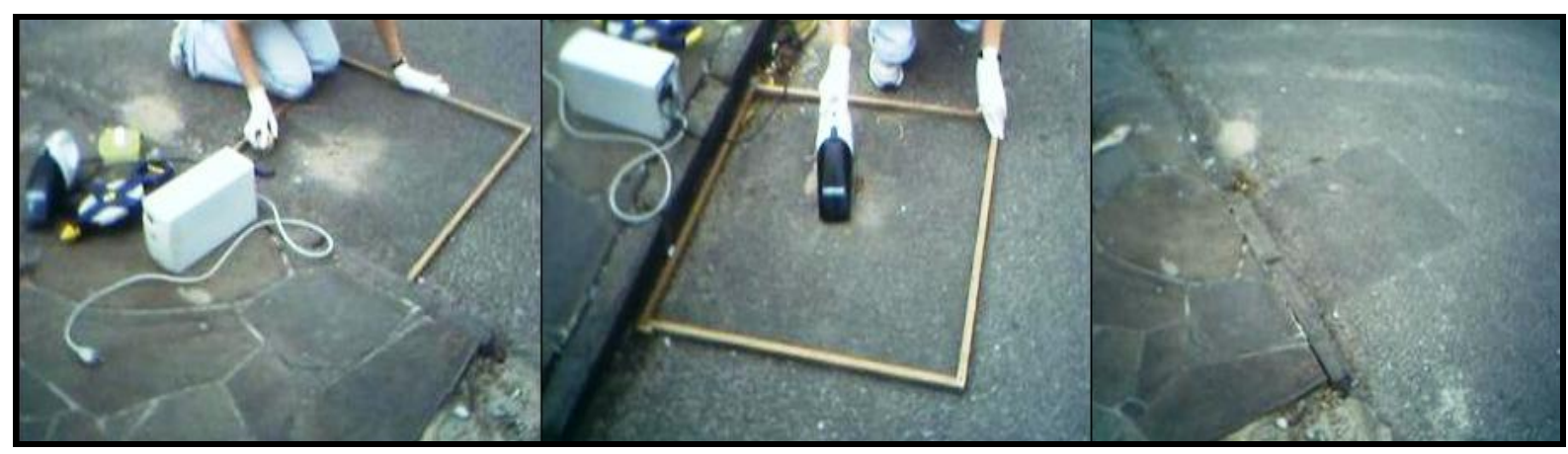

(a)

(b)

(c)

Figura 2 - Área de amostragem isolada por moldura de madeira (a) escovada; (b) aspirada; (c) depois do procedimento.

ríodos, entre eventos de precipitação, através da utilização de um aspirador de pó conforme estudos já realizados (BUTLER et al., 1992; DELETIC et al., 2000; MUTHUKARUPPAN et al., 2002; VAZE E CHIEW, 2002; DOTTO, 2006 e GOMES, 2008). O primeiro período de coleta ocorreu de 23 de julho a 01 de agosto, o segundo, de 22 a 28 de outubro, e o terceiro, de 05 a 13 de novembro de 2007.

As áreas de estudo foram dividas em subáreas e a cada dia, aproximadamente no mesmo horário, às 10:00 horas, as coletas foram realizadas em superfícies de $0,5 \mathrm{~m}^{2}(0,707 \mathrm{~m}$ x 0,707 m) escolhidas por sorteio. As superfícies só eram aspiradas novamente, se sorteadas, após evento de precipitação. A superfície amostrada foi delimitada e isolada com moldura de madeira, conforme figura 2. A superfície era escovada com escova de cerdas de nylon e a seguir aspirada com aspirador para carro de 140W.

As coletas na rua Marquês do Herval foram realizadas na primeira quadra, de 163,26 m de comprimento e $0,0531 \mathrm{~m} . \mathrm{m}^{-1}$ de declividade média. $\mathrm{Na}$ rua Rigoberto Duarte as coletas realizaram-se nas suas quatro quadras, que possuem comprimentos de 62,2, 79,46, 68,93 e 222,49 m; e declividades médias respectivas de 0,0767, 0,0960, 0,1204 e 0,1011 m.m $\mathrm{m}^{-1}$.

\section{Amostragem dos sedimentos em suspensão}

Foram realizadas coletas de amostras de sedimento em suspensão durante três eventos de precipitação, nos dias 02 de agosto, 29 de outubro e 13 e 14 de novembro de 2007. Utilizou-se um amostrador de água automático modelo ISCO, instalado junto à calha, para coletar a mistura água e sedimento na saída da canalização.

\section{Preparo das amostras de sedimento seco}

As amostras foram pesadas, retirados e pesados o material orgânico e o lixo. Após foram peneiradas nas peneiras de malha $500 \mu \mathrm{m}, 250 \mu \mathrm{m}$ e $63 \mu \mathrm{m}$. O material retido nestas peneiras e o passante na peneira $63 \mu \mathrm{m}$ foram pesados e retirados $2 \mathrm{~g}$ do material de cada granulometria para a dissolução em 100 mL de água de Milli-Q. As amostras foram agitadas para dissolução dos poluentes agregados aos sedimentos e deixadas em repouso por duas horas. Após o descanso retirou-se a parte sobrenadante e congelou-se em recipientes plásticos descontaminados, conforme metodologia proposta por Vaze e Chiew (2004).

No final de cada período de coleta de sedimento seco, as amostras foram descongeladas e agrupadas, por granulometria, para formar amostras representativas e analisadas.

\section{Preparo das amostras de sedimento em suspensão}

Após cada evento de precipitação as amostras foram retiradas do amostrador automático, misturadas, a fim de obter uma amostra representativa do evento. Retirava-se duas amostras de $1 \mathrm{~L}$ cada. Cada amostra de 1L foi passada na paneira de $1000 \mu \mathrm{m}$ e retirado $100 \mathrm{~mL}$, os $900 \mathrm{~mL}$ restantes foram passados na peneira de $500 \mu \mathrm{m}$ e retirado $100 \mathrm{~mL}$, repetindose para as peneiras de $250 \mu \mathrm{m}$ e $63 \mu \mathrm{m}$, conforme metodologia de Vaze e Chiew (2004).

\section{Parâmetros de qualidade da água analisados}

Foram determinados os seguintes parâmetros: sólidos totais, demanda química de oxigênio, 
amônia, nitrito, nitrato, fosfato, cobre, níquel, zinco e chumbo. O quadro 1 mostra os parâmetros monitorados e técnicas analíticas utilizadas.

Quadro 1 - Parâmetros e técnicas analíticas utilizadas (APHA, 1998).

\begin{tabular}{ll}
\hline Parâmetros & Técnica analítica \\
\hline Sólidos & Gravimétrico \\
\hline DQO & Titulométrico - refluxo fechado \\
\hline Amônia, Nitrito, & Cromatografia iônica \\
Nitrato, Fosfato & \\
\hline Cobre, Níquel & ICP-MS \\
\hline Zinco, Chumbo & ICP-OES \\
\hline
\end{tabular}

\section{Avaliação da carga média anual de poluentes}

A carga média de poluentes por área e tempo das ruas estudadas foi avaliada pela equação (1).

$\mathrm{L}=\mathrm{C} \cdot 10^{9} \cdot \mathrm{V} \frac{1}{\mathrm{~A} . \mathrm{T}}$

onde: L é a carga média de metais $\left(\mathrm{Kg}_{\mathrm{h}} \mathrm{ha}^{-1} \cdot \mathrm{dia}^{-1}\right)$; C é a concentração de poluentes ( $\mu$ g. $\left.\mathrm{L}^{-1}\right)$; A é a área total amostrada no período (ha); $\mathrm{V}$ é o volume total amostrado no período (L); T é o período de tempo total amostrado (dias).

Para as amostras de sedimentos no período seco, foi considerada a área total coletada em cada campanha de amostragem, o volume de amostragem após agitação e o tempo de amostragem, em cada campanha, em dias, período entre eventos de precipitação. Para as amostras de sedimento em suspensão foi considerada a área de contribuição da bacia, 1,2 ha, o volume de água pluvial amostrado e o período de tempo sem precipitação. Posteriormente foi calculada a carga média das campanhas e estimada a carga anual.

\section{Fluxo de veículos}

A acumulação de poluentes nas superfícies impermeáveis depende de vários fatores, um deles é o tráfego de veículos. Foi feita a contagem dos veículos nas ruas durante uma semana. As ruas possuem sentido duplo de tráfego. Para o cálculo do fluxo de veículos foi utilizado o quadro 2 que mostra os fatores médios de equivalência de diversos tipos de veículos.

Segundo Ferrari (1991) o período compreendido entre $6 \mathrm{~h}$ e $22 \mathrm{~h}$, num total de 16 horas, cor- responde de 80 a $90 \%$ do fluxo diário de veículos em uma rodovia. O maior fluxo foi de veículos leves, carros e motos. A rua Marquês do Herval resultou um fluxo médio de 1630 veículos por dia, e a rua Rigoberto Duarte 2533 veículos por dia.

Quadro 2 - Equivalência de veículos para vias urbanas

\begin{tabular}{ll}
\hline Tipo de veículo & Vias urbanas \\
\hline Automóveis & 1,00 \\
Caminhões médios & 1,75 \\
Caminhões pesados & 2,50 \\
Ônibus & 3,00 \\
Motocicletas & 0,75 \\
Bicicletas & 0,33 \\
\hline
\end{tabular}

Fonte: Research on Road traffic-Road Research Laboratory Londres (1965 apud Ferrari,1991)

\section{RESULTADOS E DISCUSSÕES}

\section{Carga de sedimentos secos}

A tabela 1 mostra a carga de sedimentos secos nas ruas Marquês do Herval (MH) e Rigoberto Duarte (RD), nas três campanhas de monitoramento, nas faixas granulométricas $\geq 500 \mu \mathrm{m}, \quad 500-250 \mu \mathrm{m}, \quad 250$ $63 \mu \mathrm{m},<63 \mu \mathrm{m}$ e a carga de matéria orgânica (MO) e lixo (LX) encontrado nas duas ruas.

As ruas amostradas apresentaram cargas de sedimento na mesma faixa de valores apresentado por Dotto (2006) e Gomes (2008) e cargas maiores que as encontradas por Vaze e Chiew (2002), o que pode ser influenciado pelo fato da última apresentar limpeza periódica, enquanto as demais não possuíam varrição, apenas coleta de resíduos sólidos.

A rua Marquês do Herval apresentou maior carga de sedimento na faixa granulométrica de 500$250 \mu \mathrm{m}$ em todas campanhas. A granulometria $\leq 63 \mu \mathrm{m}$ representa em média 2,5\% da carga total de sedimentos da rua Marquês do Herval e 3,6\% da rua Rigoberto Duarte. A granulometria $>250 \mu \mathrm{m}$ representa em média $69 \%$ da carga de sedimentos.

\section{Carga de poluição dos sedimentos secos}

Para cada faixa granulométrica foi avaliada a carga de poluição orgânica, de nutrientes e de metais pesados, através da determinação das concentrações dos seguintes parâmetros: demanda química de oxigênio, amônia, nitrito, nitrato, fosfato, cobre, níquel, zinco e chumbo. 
Tabela 1 - Carga de sedimentos secos por granulometria, rua e campanha.

\begin{tabular}{|c|c|c|c|c|c|c|c|c|c|c|}
\hline \multirow{3}{*}{ Granulometria } & \multicolumn{4}{|c|}{ Campanha 1} & \multicolumn{4}{|c|}{ Campanha 2} & \multirow{2}{*}{\multicolumn{2}{|c|}{$\begin{array}{c}\text { Campanha } 3 \\
\text { M.H. }\end{array}$}} \\
\hline & \multicolumn{2}{|c|}{ M.H. } & \multicolumn{2}{|c|}{ R.D. } & \multicolumn{2}{|c|}{ M.H. } & \multicolumn{2}{|c|}{ R.D. } & & \\
\hline & g.m $\mathrm{m}^{-2}$ & $\%$ & g.m. $\mathrm{m}^{-2}$ & $\%$ & g.m $\mathrm{m}^{-2}$ & $\%$ & g.m $\mathrm{m}^{-2}$ & $\%$ & g.m ${ }^{-2}$ & $\%$ \\
\hline$\geq 500 \mu \mathrm{m}$ & 26,67 & 31,5 & 14,56 & 37,1 & 29,97 & 31,6 & 38,40 & 38,5 & 22,47 & 29,2 \\
\hline $500-250 \mu \mathrm{m}$ & 31,28 & 36,9 & 10,34 & 26,4 & 35,48 & 37,5 & 30,13 & 30,2 & 33,70 & 43,9 \\
\hline $250-63 \mu \mathrm{m}$ & 22,82 & 26,9 & 10,76 & 27,4 & 23,30 & 24,6 & 25,16 & 25,2 & 16,82 & 21,9 \\
\hline$\leq 63 \mu \mathrm{m}$ & 3,03 & 3,6 & 1,56 & 4,0 & 1,89 & 2,0 & 3,25 & 3,3 & 1,57 & 2,0 \\
\hline Total sedimento & 83,80 & 98,9 & 37,22 & 94,9 & 90,64 & 95,7 & 96,94 & 97,2 & 74,59 & 97,0 \\
\hline Matéria orgânica & 0,93 & 1,1 & 2,00 & 5,1 & 1,66 & 1,7 & 2,42 & 2,4 & 1,65 & 2,2 \\
\hline Lixo & & & & & 2,43 & 2,6 & 0,43 & 0,4 & 0,63 & 0,8 \\
\hline TOTAL & 84,73 & 100,0 & 39,22 & 100,0 & 94,73 & 100,0 & 99,79 & 100,0 & 76,84 & 100,0 \\
\hline
\end{tabular}

A figura 3 mostra as cargas de DQO, amônia, nitrato e fosfato por metro quadrado nas ruas Rigoberto Duarte (RD) e Marquês do Herval (MH), nas campanhas (C1, C2 e C3). A carga de DQO foi maior na segunda campanha para as duas ruas, devido à maior carga de sedimentos, tabela 1 . Nas demais campanhas, ocorreu pequena variação da DQO, sendo mais alta na granulometria $\geq 500 \mu \mathrm{m}$.

As cargas de amônia foram maiores na faixa granulométrica de 63-250 $\mu \mathrm{m}$, com exceção das amostras coletadas na rua Marquês do Herval, terceira campanha, que obteve maior carga na faixa de 250$500 \mu \mathrm{m}$. A rua Rigoberto Duarte apresentou maior carga de amônia na campanha 2, devido a maior carga de matéria orgânica (folhas e excrementos de animais). Os valores encontrados para amônia foram, em média, menores que os encontrados por Deletic e Orr (2005), exceto os segunda campanha, que se aproximaram da carga média encontrada pelos autores, de $0,83 \mathrm{mg} \cdot \mathrm{m}^{-2}$. Não foi detectado nitrito em nenhuma das amostras.

As maiores cargas de nitrato ocorreram na faixa granulométrica de $63-250 \mu \mathrm{m}$, como observado para a amônia. Não foi detectado nitrato nas amostras com granulometria $>250 \mu \mathrm{m}$. Os valores encontrados para nitrato foram menores do que os encontrados por Deletic e Orr (2005) que encontraram uma carga média de 45,52 mg.m $\mathrm{m}^{-2}$.

As maiores cargas de fosfato foram detectadas na granulometria $\geq 500 \mu \mathrm{m}$, com exceção das amostras da segunda campanha da rua Rigoberto Duarte, que obteve maior carga na granulometria $\leq 63 \mu \mathrm{m}$. Não foi detectado fosfato nas granulometrias maiores, nem na primeira campanha de amostragem. Os valores encontrados para fosfato foram semelhantes aos encontrados por Deletic e Orr (2005), que encontraram 0,419mg. $\mathrm{m}^{-2}$.
A figura 4 mostra as cargas de cobre, zinco, níquel e chumbo detectadas nas amostras de sedimento seco coletadas nas ruas Marquês do Herval $(\mathrm{MH})$ e Rigoberto Duarte (RD) nas três campanhas de monitoramento (C1, C2 e C3).

A rua Marquês do Herval apresentou maior carga de cobre, zinco e chumbo comparada a Rigoberto Duarte, devido ao maior acúmulo de sedimentos, proporcionado pela menor declividade.

A granulometria $\leq 63 \mu \mathrm{m}$ apresentou menores cargas de metais, devido a menor carga de sedimentos na faixa granulométrica $(2,5 \%$ da carga total).

As cargas de metais pesados encontradas foram inferiores as encontradas por Deletic e Orr (2005), 49,7, 34,3 e 47,6 mg.m ${ }^{-2}$, para zinco, cobre e chumbo respectivamente.

\section{Avaliação da concentração de metais pesados nos sedimentos secos}

A tabela 2 mostra a concentração de metais pesados nos sedimentos nas ruas Rigoberto Duarte (RD) e Marquês do Herval (MH), nas três campanhas de amostragem (C1, C2 e C3).

Todos os metais apresentaram o mesmo comportamento, maiores concentrações na granulometria $\leq 63 \mu \mathrm{m}$, comprovando estudos anteriores (DELETIC E ORR, 2005 e POLETO et al., 2008) que afirmam que os metais aderem-se as menores partículas. O comportamento da carga difere do comportamento da concentração, porque a carga de metais depende da carga de sedimentos, e a concentração independe.

As concentrações de metais foram inferiores as encontradas por Ellis e Revitt (1982), que encontraram concentrações de 280,695 e $1826 \mu \mathrm{g} \cdot \mathrm{g}^{-1}$ para 

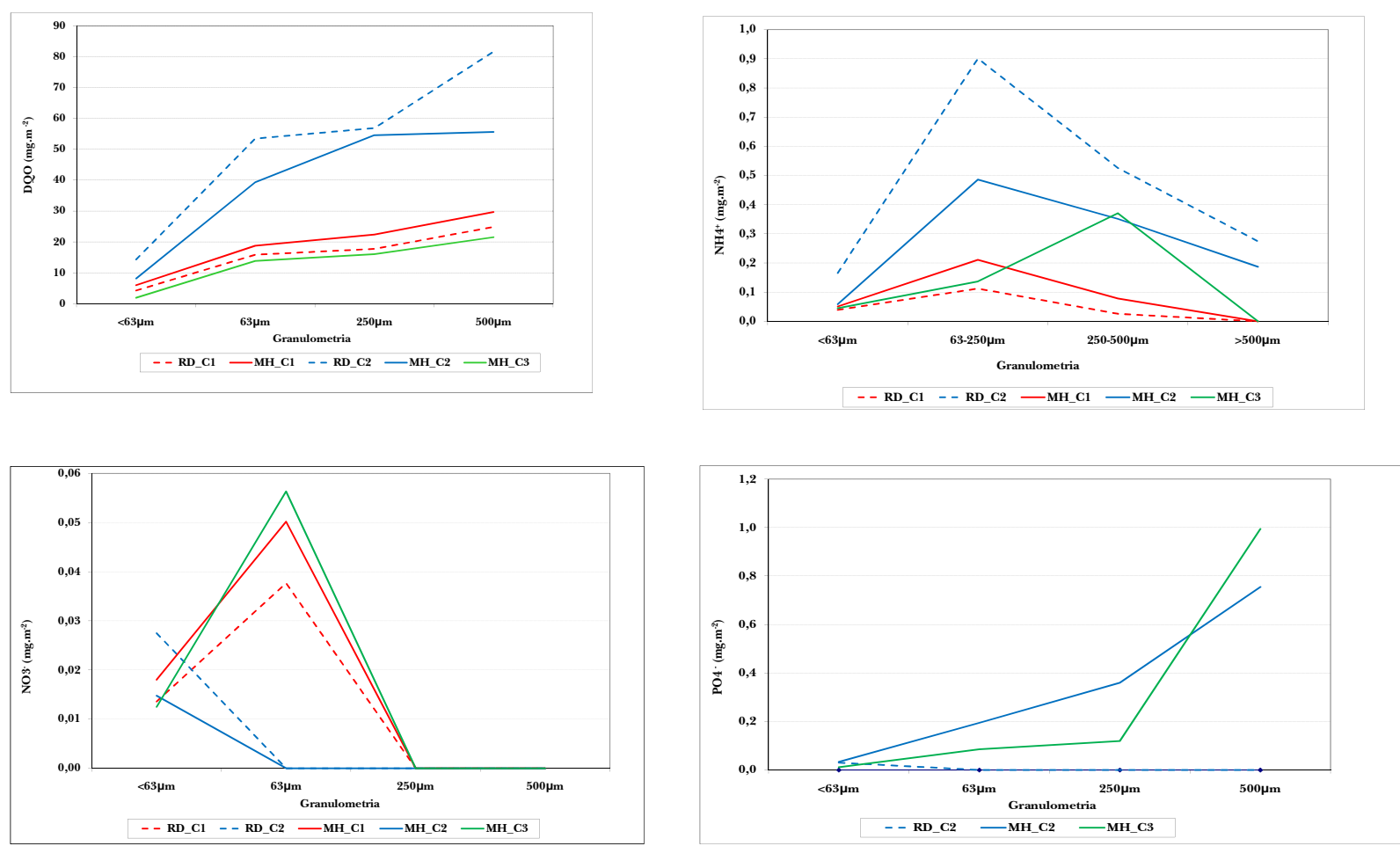

Figura 3 - Cargas de DQO, $\mathrm{NH}_{4}^{+}, \mathrm{NO}_{3}^{-}, \mathrm{PO}_{4}^{-}$nas ruas Marquês do Herval (MH) e Rigoberto Duarte (RD) nas três campanhas de amostragem $(\mathrm{C} 1, \mathrm{C} 2, \mathrm{C} 3)$.
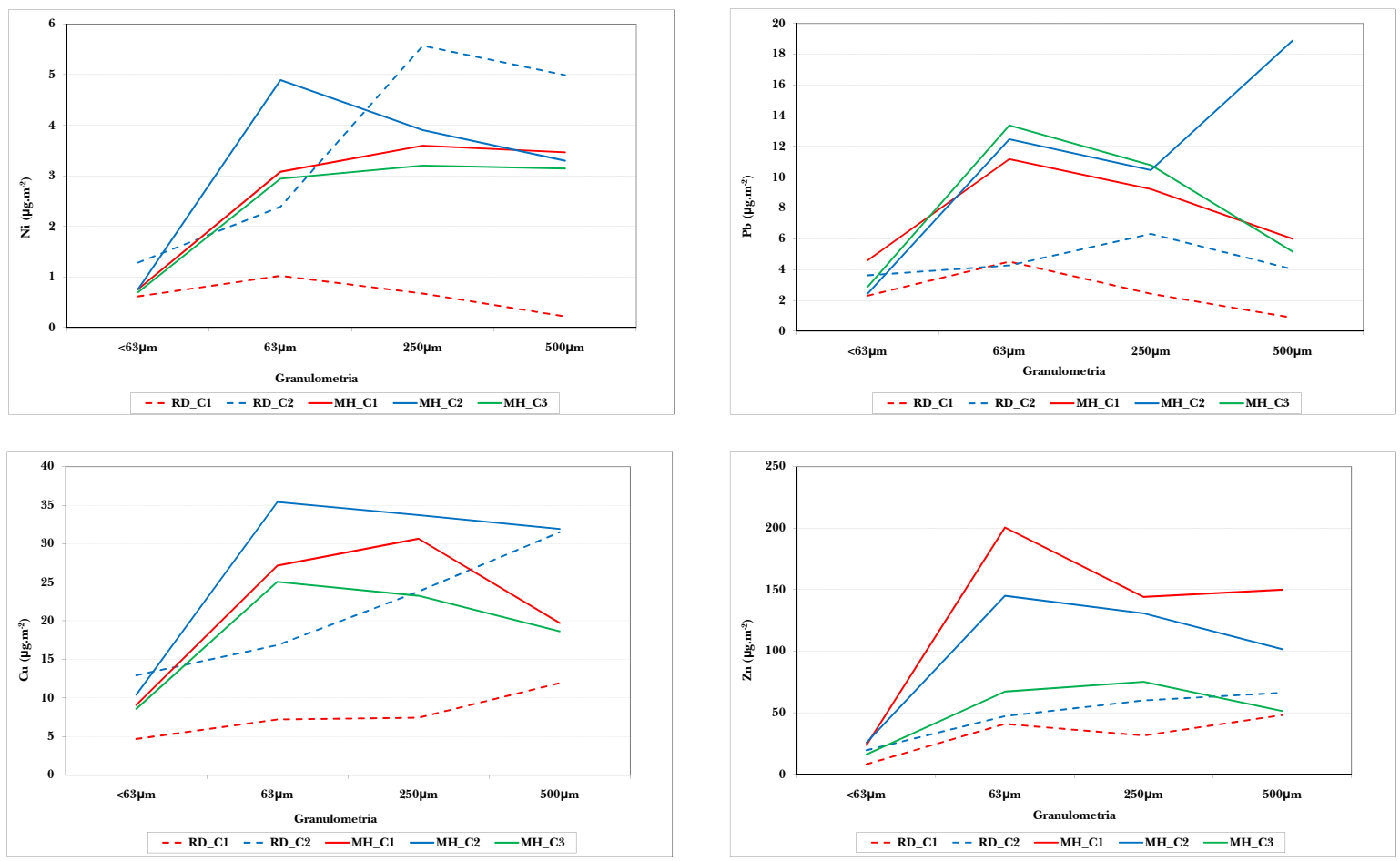

Figura 4 - Cargas de cobre ( $\mathrm{Cu}$ ), zinco $(\mathrm{Zn})$, níquel $(\mathrm{Ni})$ e chumbo $(\mathrm{Pb})$ nas ruas Marques do Herval $(\mathrm{MH})$ e Rigoberto Duarte (RD) nas campanhas de amostragem (C1, C2 e C3) 
Tabela 2 - Concentrações de metais pesados nas ruas Rigoberto Duarte (RD) e Marquês do Herval (MH), nas campanhas de monitoramento (C1, C2 e C3) e faixas granulométricas.

\begin{tabular}{|l|l|c|c|c|c|c|}
\hline & & \multicolumn{5}{|c|}{${\text { Concentraçóes }\left(\boldsymbol{\mu g} \cdot \mathbf{g}^{-1}\right)}$} \\
\hline Metais & Granulometria & RD C1 & MH C1 & RD C2 & MH C2 & MH C3 \\
\hline \multirow{4}{*}{ Cu } & $\geq 500 \mu \mathrm{m}$ & 0,820 & 0,740 & 0,820 & 1,065 & 0,830 \\
\cline { 2 - 7 } & $500-250 \mu \mathrm{m}$ & 0,720 & 0,980 & 0,790 & 0,950 & 0,690 \\
\cline { 2 - 7 } & $250-63 \mu \mathrm{m}$ & 0,670 & 1,190 & 0,670 & 1,520 & 1,490 \\
\cline { 2 - 7 } & $\leq 63 \mu \mathrm{m}$ & 3,005 & 3,005 & 3,980 & 5,510 & 5,475 \\
\hline \multirow{4}{*}{$\mathbf{Z n}$} & $\geq 500 \mu \mathrm{m}$ & 3,300 & 5,620 & 1,725 & 3,390 & 2,285 \\
\cline { 2 - 7 } & $500-250 \mu \mathrm{m}$ & 3,045 & 4,605 & 1,990 & 3,685 & 2,230 \\
\cline { 2 - 7 } & $250-63 \mu \mathrm{m}$ & 3,800 & 8,790 & 1,875 & 6,225 & 3,995 \\
\cline { 2 - 7 } & $\leq 63 \mu \mathrm{m}$ & 5,220 & 7,820 & 5,980 & 13,555 & 10,215 \\
\hline \multirow{4}{*}{ Ni } & $\geq 500 \mu \mathrm{m}$ & 0,015 & 0,130 & 0,130 & 0,110 & 0,140 \\
\cline { 2 - 7 } & $500-250 \mu \mathrm{m}$ & 0,065 & 0,115 & 0,185 & 0,110 & 0,095 \\
\cline { 2 - 7 } & $250-63 \mu \mathrm{m}$ & 0,095 & 0,135 & 0,095 & 0,210 & 0,175 \\
\cline { 2 - 7 } & $\leq 63 \mu \mathrm{m}$ & 0,395 & 0,250 & 0,395 & 0,400 & 0,445 \\
\hline \multirow{4}{*}{$\mathbf{P b}$} & $\geq 500 \mu \mathrm{m}$ & 0,060 & 0,225 & 0,105 & 0,630 & 0,230 \\
\cline { 2 - 7 } & $500-250 \mu \mathrm{m}$ & 0,235 & 0,295 & 0,210 & 0,295 & 0,320 \\
\cline { 2 - 7 } & $250-63 \mu \mathrm{m}$ & 0,420 & 0,490 & 0,170 & 0,535 & 0,795 \\
\cline { 2 - 7 } & $\leq 63 \mu \mathrm{m}$ & 1,480 & 1,525 & 1,115 & 1,295 & 1,840 \\
\hline
\end{tabular}

Tabela 3 - Características dos eventos monitorados

\begin{tabular}{|l|c|c|c|c|c|}
\hline Data & Duração & $\begin{array}{c}\text { Total } \\
\text { precipitado } \\
(\mathbf{m m})\end{array}$ & $\begin{array}{c}\text { Vazão média } \\
(\mathbf{L} / \mathbf{s})\end{array}$ & $\begin{array}{c}\text { Período de tempo seco } \\
\text { antecedente ao evento } \\
\text { (dias) }\end{array}$ & $\begin{array}{c}\text { Volume } \\
\text { escoado } \\
(\mathbf{L})\end{array}$ \\
\hline $02 / 08 / 2007$ & $1 \mathrm{~h} 21 \mathrm{~min}$ & 4,88 & 8,89 & 10 & 43.760 \\
\hline $29 / 10 / 2007$ & $14 \mathrm{~h} 16 \mathrm{~min}$ & 62,81 & 12,59 & 7 & 646.761 \\
\hline $13 / 11 / 2007$ & $5 \mathrm{~h} 30 \mathrm{~min}$ & 15,75 & 3,37 & 9 & 66.867 \\
\hline
\end{tabular}

cobre, zinco e chumbo respectivamente, na granulometria $<250 \mu \mathrm{m}$ e concentrações de 110,245 e 723 $\mu \mathrm{g} \cdot \mathrm{g}^{-1}$ para a granulometria $>250 \mu \mathrm{m}$. Deletic e Orr (2005) encontraram concentrações de cobre, zinco e chumbo, de 530, 1100 e $1900 \mu \mathrm{g} \cdot \mathrm{g}^{-1}$ para a granulometria $\leq 63 \mu \mathrm{m}$. Esta diferença pode ser devido às ruas amostradas possuírem menor tráfego de veículos e possuírem ocupação residencial diferente das da bibliografia.

As maiores concentrações de zinco foram detectadas nas amostras de sedimento da rua Marquês do Herval, devido a existir uma oficina mecânica neste local.

O zinco foi o metal pesado com maior concentração encontrada nas amostras, seguido por cobre e chumbo, essa tendência também foi observada por Push et al. (2007).

\section{Avaliação da carga de poluição na microdrenagem}

A tabela 3 mostra as características dos eventos analisados.

A tabela 4 mostra a carga de DQO, nutrientes e metais pesados nas faixas granulométricas do escoamento pluvial, para as três campanhas de monitoramento.

As maiores concentrações de sólidos, DQO, nutrientes e metais pesados ocorreram na segunda campanha, devido a uma obra realizada na rede de microdrenagem, pela maior precipitação e elevada vazão, obtendo maior força para carrear os sedimentos.

As cargas de nutrientes apresentaram pouca variabilidade nas diferentes faixas granulométricas analisadas, na primeira e na segunda campanha de amostragem. 
Tabela 4 - Cargas de poluentes no ES nas 4 faixas granulométricas

\begin{tabular}{|c|c|c|c|c|}
\hline & Granulometria & ES C1 & ES C2 & ES C3 \\
\hline \multirow{4}{*}{ DQO (mg.m $\left.{ }^{-2}\right)$} & $\geq 500 \mu \mathrm{m}$ & 91 & 2239 & 57 \\
\hline & $500-250 \mu \mathrm{m}$ & 84 & 1659 & 66 \\
\hline & $250-63 \mu \mathrm{m}$ & 86 & 1372 & 62 \\
\hline & $\leq 63 \mu \mathrm{m}$ & 79 & 1284 & 56 \\
\hline \multirow{4}{*}{ ST $\left(\mathbf{m g} \cdot \mathbf{m}^{-2}\right)$} & $\geq 500 \mu \mathrm{m}$ & 182 & 16367 & 258 \\
\hline & $500-250 \mu \mathrm{m}$ & 166 & 14506 & 208 \\
\hline & $250-63 \mu \mathrm{m}$ & 219 & 14948 & 503 \\
\hline & $<63 \mu \mathrm{m}$ & 252 & 12236 & 149 \\
\hline \multirow{4}{*}{$\mathrm{NH}_{4}^{+}\left(\mathbf{m g} \cdot \mathbf{m}^{-2}\right)$} & $\geq 500 \mu \mathrm{m}$ & 0,317 & 7,963 & n.d \\
\hline & $500-250 \mu \mathrm{m}$ & 0,168 & 6,938 & 0,101 \\
\hline & $250-63 \mu \mathrm{m}$ & 0,377 & 9,098 & 0,117 \\
\hline & $\leq 63 \mu \mathrm{m}$ & 0,346 & 6,559 & 0,169 \\
\hline \multirow{4}{*}{$\mathrm{NO}_{3}^{-}\left(\mathbf{m g} \cdot \mathbf{m}^{-2}\right)$} & $\geq 500 \mu \mathrm{m}$ & 1,405 & 8,310 & 0,536 \\
\hline & $500-250 \mu \mathrm{m}$ & 1,444 & 8,530 & 0,536 \\
\hline & $250-63 \mu \mathrm{m}$ & 1,136 & 8,814 & 0,522 \\
\hline & $\leq 63 \mu \mathrm{m}$ & 1,444 & 8,751 & 0,544 \\
\hline \multirow{4}{*}{$\mathrm{PO}_{4}^{-}\left(\mathrm{mg} \cdot \mathrm{m}^{-2}\right)$} & $\geq 500 \mu \mathrm{m}$ & n.d & n.d & 0,293 \\
\hline & $500-250 \mu \mathrm{m}$ & n.d & n.d & 0,306 \\
\hline & $250-63 \mu \mathrm{m}$ & n.d & n.d & 0,286 \\
\hline & $\leq 63 \mu \mathrm{m}$ & n.d & 2,649 & 0,289 \\
\hline \multirow{4}{*}{$\mathrm{Cu}\left(\boldsymbol{\mu g} \cdot \mathbf{m}^{-2}\right)$} & $\geq 500 \mu \mathrm{m}$ & 10,138 & 793,122 & 24,471 \\
\hline & $500-250 \mu \mathrm{m}$ & 13,448 & 1122,670 & 32,408 \\
\hline & $250-63 \mu \mathrm{m}$ & 10,552 & 1121,093 & 39,353 \\
\hline & $\leq 63 \mu \mathrm{m}$ & 15,310 & 1070,636 & 6,614 \\
\hline \multirow{4}{*}{$\mathrm{Zn}\left(\boldsymbol{\mu g} \cdot \mathbf{m}^{-2}\right)$} & $\geq 500 \mu \mathrm{m}$ & 171,309 & 2511,817 & 148,316 \\
\hline & $500-250 \mu \mathrm{m}$ & 190,964 & 4046,027 & 152,615 \\
\hline & $250-63 \mu \mathrm{m}$ & 277,860 & 4194,245 & 130,293 \\
\hline & $\leq 63 \mu \mathrm{m}$ & 426,617 & 3993,993 & 131,120 \\
\hline \multirow{4}{*}{$\mathrm{Ni}\left(\boldsymbol{\mu g} \cdot \mathbf{m}^{-2}\right)$} & $\geq 500 \mu \mathrm{m}$ & 8,896 & 263,323 & 5,456 \\
\hline & $500-250 \mu \mathrm{m}$ & 9,931 & 391,042 & 4,630 \\
\hline & $250-63 \mu \mathrm{m}$ & 10,759 & 398,926 & 3,307 \\
\hline & $\leq 63 \mu \mathrm{m}$ & 10,138 & 397,350 & 2,976 \\
\hline \multirow{4}{*}{$\mathrm{Pb}\left(\boldsymbol{\mu g} \cdot \mathrm{m}^{-2}\right)$} & $\geq 500 \mu \mathrm{m}$ & 170,895 & 392,619 & 11,574 \\
\hline & $500-250 \mu \mathrm{m}$ & 158,688 & 403,657 & 13,062 \\
\hline & $250-63 \mu \mathrm{m}$ & 497,996 & 753,703 & 15,212 \\
\hline & $\leq 63 \mu \mathrm{m}$ & 740,890 & 742,665 & 10,252 \\
\hline
\end{tabular}

A amônia apresentou picos na faixa granulométrica de 63 - $250 \mu \mathrm{m}$, comportamento semelhante ao obtido nas amostras de sedimento seco.

O fosfato, nas amostras de sedimento em suspensão, apresentou comportamento oposto aos da amostra de sedimento seco, ou seja, maior carga na granulometria menor ou igual que $63 \mu \mathrm{m}$.

As amostras do escoamento superficial na primeira campanha apresentaram maiores cargas de cobre, chumbo e zinco na granulometria $\leq 63 \mu \mathrm{m}$. As amostras de sedimento seco apresentaram maiores carga de chumbo e zinco na faixa granulométrica de 250-63 $\mu \mathrm{m}$. Este comportamento se explica por que a carga de poluentes está correlacionada com a quantidade de sólidos encontrada nas amostras. A primeira campanha apresentou maior carga de sólidos totais na menor granulometria influenciando assim a carga de metais pesados. 
As menores cargas de cobre, zinco e níquel foram obtidas na granulometria $\geq 500 \mu \mathrm{m}$ nas amostras da primeira e da segunda campanha.

Estimativa da carga média anual de poluentes nas amostras secas e do escoamento pluvial

A tabela 5 mostra uma estimativa da carga média anual do sedimento depositado na rua Marquês do Herval (MH) e do escoamento superficial (ES).

A carga média anual estimada para o escoamento superficial é superior a do sedimento depositado na rua, sendo até 3400 vezes maior para nitrato e 1030 para chumbo. Os valores encontrados para a carga anual dos metais pesados encontrados na superfície de rua são menores do que os encontrados por Push et al. (2007), que encontrou para $\mathrm{Cu}$, $\mathrm{Pb}$ e Zn valores de 0,106, 0,128 e 3,34 Kg.ha ${ }^{-1} \cdot$ ano $^{-1}$, respectivamente.

É difícil dizer que os poluentes encontrados no escoamento superficial sejam provenientes somente do material depositado na rua Marquês do Herval. Esta diferença deve-se ao fato do carreamento de poluentes provenientes de ruas adjacentes e da existência de ligações clandestinas de esgoto doméstico na canalização pluvial.

Tabela 5 - Carga média anual de poluentes na rua M.H. e no escoamento superficial (ES)

\begin{tabular}{|l|c|c|}
\hline Parâmetro & \multicolumn{2}{|c|}{ Carga $\left(\right.$ Kg.ha $^{-1}$.ano $^{-1}$ ) } \\
\hline & M.H. & ES \\
\hline $\mathbf{C u}$ & $4,77 \mathrm{E}-04$ & $1,98 \mathrm{E}-01$ \\
\hline $\mathbf{~ N i}$ & $1,50 \mathrm{E}-03$ & $7,62 \mathrm{E}-01$ \\
\hline $\mathbf{P b}$ & $4,75 \mathrm{E}-05$ & $7,01 \mathrm{E}-02$ \\
\hline $\mathbf{D Q O}$ & $1,76 \mathrm{E}-04$ & $1,82 \mathrm{E}-01$ \\
\hline NH$_{4}{ }^{+}$ & $5,78 \mathrm{E}-01$ & $3,32 \mathrm{E}+02$ \\
\hline $\mathbf{N O}_{3}^{-}$ & $2,87 \mathrm{E}-03$ & $1,50 \mathrm{E}+00$ \\
\hline PO $_{4}^{-}$ & $5,71 \mathrm{E}-04$ & $1,95 \mathrm{E}+00$ \\
\hline
\end{tabular}

\section{CONCLUSÕES}

Quantificaram-se poluentes ligados aos diversos tamanhos de sedimentos em superfícies impermeáveis de ruas com intensidade de urbanização semelhante, porém com intensidade de tráfego e declividades diferentes. Foram coletados sedimentos secos nas ruas Marquês do Herval e Rigoberto Duarte; e monitoramento hidrossedimentológico na saída da canalização da rua Marquês do Herval.

A rua Marquês do Herval apresentou uma maior carga de sedimentos, maior quantidade de material fino e maior carga de metais comparado com a rua Rigoberto Duarte, por possuir menor inclinação, embora com menor fluxo médio de veículos.

A granulometria $\leq 63 \mu \mathrm{m}$ representa em média $3 \%$ da carga total de sedimentos e a granulometria $>250 \mu \mathrm{m}$ representa em média $69 \%$ da carga de sedimentos nas ruas.

Nas amostras de sedimento seco, as maiores cargas de DQO e fosfato foram encontradas na granulometria $\geq 500 \mu \mathrm{m}$. A amônia e o nitrato, chumbo e zinco estão associados a faixa granulométrica de $63-250 \mu \mathrm{m}$.

As concentrações de metais pesados apresentaram o mesmo comportamento, maiores concentrações na granulometria $\leq 63 \mu \mathrm{m}$, comprovando estudos realizados por Deletic e Orr (2005) e Egotawatta e Goonetilleke (2006) que constataram que os metais aderem-se as menores partículas.

As amostras coletadas no escoamento superficial apresentaram grandes diferenças de cargas de sólidos dependendo das campanhas, devido à realização de obras e variabilidade das características da precipitação. Quanto maior a intensidade da precipitação e vazão maior o carreamento dos sedimentos.

As cargas de nutrientes apresentaram pouca variabilidade nas diferentes faixas granulométricas analisadas, na primeira e na segunda campanha de amostragem do escoamento superficial. A amônia e o sulfato apresentaram picos na faixa granulométrica de $63-250 \mu \mathrm{m}$, comportamento semelhante ao obtido nas amostras de sedimento seco.

Foram estimadas cargas de poluentes para comparar a quantidade de poluentes encontrada nas amostras de sedimento seco coletados na rua Marquês do Herval e a quantidade de poluentes encontradas nas amostras do escoamento pluvial proveniente da microdrenagem. Constatou-se que a carga média anual estimada para o escoamento superficial superior a carga média anual estimada para o sedimento depositado na rua, provavelmente devido ao carreamento pelo escoamento superficial de poluentes provenientes de ruas adjacentes e também pelo fato de que há ligações clandestinas de esgoto doméstico na canalização pluvial. 


\section{AGRADECIMENTOS}

Ao CT-Hidro e ao CNPq pelas bolsas de mestrado, de pesquisa concedidas. Os autores agradecem aos técnicos de laboratório Alcides Sartóri e Thiago Augusto Formentini pelo auxílio nos trabalhos de campo e análises de água e ao Grupo GHIDROS pelo fornecimento dos dados hidrológicos.

\section{REFERÊNCIAS}

APHA, AWWA, WEF Standard Methods for the Examination of Water and Wastewater. 20.Ed., Washington DC: American Public Health Association, 1998.

BUTLER, D.; THEDCHANAMOORTHY, S.; PAYNE, J.A. Aspects of surface sediment characteristics on an urban catchment in London. Water Science Technology.v. 25, n. 8, p. 13-19, 1992.

CARVALHO, N.O. Guia de praticas sedimentométricas. Agência Nacional de Energia Elétrica, Superintendência de estudos e informações hidrológicas, 154 p. 2000.

DELETIC, A.; ASHLEY, R.; REST, D. Modelling input of fine granular sediment into drainage systems via gully-pots. Water Research. v. 34, n. 15, p. 3836-3844, 2000 .

DELETIC, A.; ORR, D. W. Pollution buildup on road surfaces. Journal of Environmental Engineering- ASCE. v. 131, n. 1, p. 391-396, 2005.

DOTTO, C. B. S. Acumulação e balanço de sedimentos em superfícies asfálticas em área urbana de Santa Maria - RS. 126 f. Dissertação (Mestrado em Engenharia Civil) Universidade Federal de Santa Maria, Santa Maria, 2006.

EGODAWATA, P.; GOONETILLEKE, A. Characteristics off pollutants built-up on residential road surface. In: PROCEEDINGS OF THE $7^{\mathrm{TH}}$ INTERNATIONAL CONFERENCE ON HYDROSCIENCE AND ENGINEERING, Philadelphia, USA, 2006.

ELLIS, J. B.; REVITT, D. M. Incidence of heavy metals in street surface sediments: solubility and grain size studies. Water, Air and Soil Pollution. v.17, p. 87-100, 1982.
FERRARI, C. Curso de planejamento municipal integrado. Ed. São Paulo: Pioneira, 1991.

GOMES, A. P. Acumulação e transporte de sedimentos na microdrenagem: monitoramento e modelagem. $146 \mathrm{f}$. Dissertação (Mestrado em Engenharia Civil). Universidade Federal de Santa Maria, Santa Maria, 2008.

MENEZES, F. L.; ROSSO, T. C. A. Avaliação da qualidade de águas de drenagem urbana correlacionada aos poluentes originados pelo tráfego de veículos automotores. In: XVII SIMPÓSIO BRASILEIRO DE RECURSOS HÍDRICOS, 2007, São Paulo. 2007.

MUTHUKARUPPAN, M.; CHIEW, F. H. S.; WONG, T. (2002) Size distribution and partitioning of urban pollutants. Global Solutions for Urban Drainage, 2002. (CD-ROM).

POLETO, C; MERTEN, G. H. Urban street pollutants. In: INTERNATIONAL CONFERENCE ON DIFFUSE POLLUTION, 11 ${ }^{\text {th }}$., 2007, Belo Horizonte. 2007.

PUSH, P. B.; GUIMARÃES, J. R.; GRASSI, M. T. Estimativa de cargas de metais a partir de fontes difusas de poluição urbana. In: XVII SIMPÓSIO BRASILEIRO DE RECURSOS HÍDRICOS, 2007, São Paulo. 2007.

VAZE, J.; CHIEW, F. H. S. Experimental study of pollutant accumulation on an urban road surface. Urban Water, v. 4, n. 4, p. 379-389, 2002.

VAZE, J.; CHIEW, F. H. S. Nutrient loads associated with different sediments sizes in urban stormwater and surface pollutants. Journal of Environmental EngineeringASCE, v. 130, n. 4, p. 391-396, 2004.

\section{Study of Pollutant Distribution on Urban Surfaces}

\section{ABSTRACT}

This paper aimed to quantify pollutants connected to sediments of different sizes on impermeable surfaces. Dry sediments were collected on two streets and hydrosedimentological monitoring was performed at the outlet of the drainage system in one of them. Dry sediment samples prior to rainfall, as well as surface runoff sediment samples during rainfall, were separated into 4 granulometric bands ( $\leq 63 \mu \mathrm{m}, 63-250 \mu \mathrm{m}, 250-500 \mu \mathrm{m}$ e $\geq 500 \mu \mathrm{m})$. Organic pollution, nutrient and heavy metal contents were evaluated for each granulometric band. Heavy metal samples showed 
higher concentrations in granulometry below $63 \mu \mathrm{m}$. In dry sediment samples, the highest chemical oxygen demand and phosphate contents were found in granulometry above


with the 63-250 um granulometric band.

Key-words: sediment, heavy metals, nutrients, nonpoint pollution. 